\title{
Article \\ Nanoparticle-Induced Property Changes in Nematic Liquid Crystals
}

\author{
Nicolas Brouckaert ${ }^{1, *} *$, Nina Podoliak ${ }^{1}$, Tetiana Orlova ${ }^{1, *}{ }^{\oplus}$, Denitsa Bankova ${ }^{1}$, Angela F. De Fazio ${ }^{2} \oplus$, \\ Antonios G. Kanaras ${ }^{1} \mathbb{D}$, Ondrej Hovorka ${ }^{3}$, Giampaolo D'Alessandro ${ }^{4, *} \mathbb{D}$ and Malgosia Kaczmarek ${ }^{1, *}$
}

Citation: Brouckaert, N.; Podoliak, N.; Orlova, T.; Bankova, D.; De Fazio, A.F.; Kanaras, A.G.; Hovorka, O.; D'Alessandro, G.; Kaczmarek, M. Nanoparticle-Induced Property Changes in Nematic Liquid Crystals. Nanomaterials 2022, 12, 341. https:// doi.org/10.3390/nano12030341

Academic Editor: Yann Molard

Received: 17 December 2021

Accepted: 18 January 2022

Published: 21 January 2022

Publisher's Note: MDPI stays neutral with regard to jurisdictional claims in published maps and institutional affiliations.

Copyright: (C) 2022 by the authors. Licensee MDPI, Basel, Switzerland. This article is an open access article distributed under the terms and conditions of the Creative Commons Attribution (CC BY) license (https:// creativecommons.org/licenses/by/ $4.0 /)$.
1 School of Physics and Astronomy, University of Southampton, Southampton S017 1BJ, UK; N.Podoliak@soton.ac.uk (N.P.); d.o.bankova@soton.ac.uk (D.B.); a.kanaras@soton.ac.uk (A.G.K.)

2 Istituto Italiano di Tecnologia, Via Morego 30, 16163 Genova, Italy; angela.defazio@iit.it

3 Faculty of Engineering and Physical Sciences, University of Southampton, Southampton S017 7QF, UK; o.hovorka@soton.ac.uk

4 School of Mathematics, University of Southampton, Southampton S017 1BJ, UK

* Correspondence: nb1f20@soton.ac.uk (N.B.); t.orlova@soton.ac.uk (T.O.); dales@soton.ac.uk (G.D.); mfk@soton.ac.uk (M.K.)

\begin{abstract}
Doping liquid crystals with nanoparticles is a widely accepted method to enhance liquid crystal's intrinsic properties. In this study, a quick and reliable method to characterise such colloidal suspensions using an optical multi-parameter analyser, a cross-polarised intensity measurementbased device, is presented. Suspensions characterised in this work are either plasmonic (azo-thiol gold AzoGNPs) or ferroelectric $\mathrm{Sn}_{2} \mathrm{P}_{2} \mathrm{~S}_{6}$ (SPS) nanoparticles in nematic liquid crystals. The elastic constants and rotational viscosity showed nonlinear dependence on the concentration of AzoGNPs, initially increasing at lower concentrations and then decreasing at higher concentrations, indicating some degree of particle aggregation. For the SPS suspension, the elastic constant decreased with doping, while the rotational viscosity increased, in agreement with previous findings. Through viscosity measurements, the stability of SPS suspension over ten years is also highlighted.
\end{abstract}

Keywords: liquid crystal; nanoparticles; colloidal suspension; characterisation; optical multi-parameter analyser; elastic constants; rotational viscosity

\section{Introduction}

The electro-optic properties of liquid crystals (LC) are widely exploited in optical devices, such as displays, light modulators and variable wave plates [1-3]. Their birefringence and dielectric anisotropy, coupled with their elastic properties, allow these materials to be quickly and reversibly addressed by an external electric or magnetic field, making liquid crystals perfect for commercial displays. However, as the general technology evolves, requirements for the liquid crystals change too. A shorter response time or lower operating voltage are common examples of LC characteristics that must be continuously improved to match the increasing demand.

A solution to avoid chemical synthesis of new types of LCs is to dope existing LCs with nanoparticles to change their properties [1-9]. A variety of nanoparticles such as ferroelectrics [10-15], gold [5,6,16-21], quantum dots [22-28], oxides [4,7,29] and magnetic nanoparticles [30-32] have been tested in LCs. It has been reported that doping nematic liquid crystals (NLC) with ferroelectric nanoparticles such as $\mathrm{Sn}_{2} \mathrm{P}_{2} \mathrm{~S}_{6}$ (SPS) or $\mathrm{BaTiO}_{3}$ (BTO) can efficiently decrease the driving voltage, enhance the dielectric response $[1,10,11]$ and increase the optical anisotropy [14]. The main mechanism behind such effects is the coupling between the natural polarisation of the nanoparticles and the LCs, increasing the molecular order and the electro-optical properties of the blend [15]. The impact of the ferroelectric nanoparticles highly depends on their concentration and size. The concentration is usually kept below $1 \mathrm{wt} \%$ to avoid significant particle aggregation, while the size is in the range of 
10 to 100 nanometres [11,12]. Smaller particles may lose their ferroelectric properties, while bigger ones can contain multiple ferroelectric domains [11,13].

Another type of nanoparticle, widely employed with LCs, are metallic nanoparticles, such as gold. These improve the electro-optical properties of LCs by, once again, increasing the molecular order of the blend [1,5,6,33]. Gold nanoparticles also possess plasmonic resonances, leading to an increase in the nonlinear optical properties of the LC [6,17-19]. Another advantage of gold nanoparticles (GNPs) is the possibility of functionalising them with different types of surfactants, including mesogenic ligands. Functionalising gold nanoparticles increases their solubility in the LC host, making it possible to obtain stable suspensions with a higher concentration $[5,21]$. It has also been demonstrated that functionalising GNPs with azo-thiol ligands can also induce a reversible change of alignment of the 5CB LC upon light irradiation, from homeotropic under UV to planar when irradiated by visible light [20].

However, doping with NPs may also lead to particle aggregation. Its impact on the LC can be either beneficial, by reducing its viscosity by generating clusters that leave more space for the LCs to move freely $[6,10]$, or detrimental, by degrading its elastic properties [8] or causing light scattering. These contrasting effects must be well understood and controlled to ensure that the suspension has the desired properties.

With the expanding research on doped LCs, there is an increasing need for a quick and reliable method of quantifying the electro-optical and physical properties of LC composites. In this study, we propose a method to characterise colloidal suspensions using an optical multi-parameter analyser (OMPA), a method originally designed for undoped LCs [34,35]. It measures critical LC properties such as elastics constants (splay elastic constant $\mathrm{K}_{1}$ and bend elastic constant $K_{3}$ ), pretilt $\theta$, anchoring energy $W_{p}$ and rotational viscosity $\gamma_{1}$ from simple cross-polarised intensity experiments (CPI). We demonstrate, for the first time, the use of OMPA to study the changes of E7 and 5CB electro-optical and viscosity properties when doped with novel nanoparticles, azo-thiol-functionalized gold nanoparticles (AzoGNPs), with the concentrations up to $3 \mathrm{wt} \%$. We also measure the properties of nematic LC18523 doped with $1 \mathrm{wt} \%$ SPS nanoparticles and test the stability over time. We compare its elastic constants and rotational viscosity from previously published results [35] and extend its characterisation by studying the impact of ageing on these cells on both elastic constants and rotational viscosity. We, therefore, demonstrate the ability of the OMPA technique to easily characterise the key properties of both pure and doped LCs.

\section{Materials and Methods}

\subsection{Particles Synthesis}

The synthesis of small gold nanoparticles functionalized with Azo-C11-SH ligands was achieved following previously published protocols [20,36]. Briefly, an aqueous solution of $\mathrm{HAuCl}_{4}(1.5 \mathrm{~mL}, 30 \mathrm{mM})$ was mixed with a solution of tetraoctylammonium bromide (TOAB) in toluene $(4 \mathrm{~mL}, 50 \mathrm{mM})$. The two-phase mixture was rapidly stirred until all $\mathrm{Au}^{3+}$ was transferred to the organic phase to produce a deep-red solution. After discarding the aqueous phase, Azo-C11-SH $(26 \mu \mathrm{mol})$ was added to the solution (Gold:Azo-C11-SH $\approx 2: 1)$. Then, $\mathrm{NaBH}_{4}(1.5 \mathrm{~mL}, 0.3 \mathrm{M})$ was slowly added under vigorous stirring. The reaction stirred for $2 \mathrm{~h}$, and the product was purified by centrifugation using ethanol $(3 \times$, $5000 \mathrm{rpm}$ ). The $\mathrm{Au}$ core of nanoparticles was $5 \mathrm{~nm}$ in diameter, as measured by transmission electron microscopy (TEM). Finally, the Azo-gold nanoparticles were stored in toluene (concentration of $2.5 \mathrm{mg} / \mathrm{mL}$ ) at $4{ }^{\circ} \mathrm{C}$ until further use.

$\mathrm{HAuCl}_{4}, \mathrm{TOAB}, \mathrm{NaBH}_{4}$ and all the solvents were purchased from Merck SigmaAldrich (Burlington, MA, USA) and used without further purification. The Azo-C11-SH ligand was purchased from Prochimia Surfaces (Gdynia, Poland).

$\mathrm{Sn}_{2} \mathrm{P}_{2} \mathrm{~S}_{6}$ (SPS) nanoparticles were prepared by milling micron-sized particles in a vibration mill. Surfactant (oleic acid) and a solvent (heptane) were added during the milling to stabilize the particle suspension. The resulting average size of nanoparticles was $50 \mathrm{~nm}$, as confirmed by atomic force microscopy (AFM) and TEM [35,37]. 


\subsection{Suspension Preparation}

Suspensions of AzoGNPs in nematic LCs E7 and 5CB, with a weight content of $1 \mathrm{wt} \%$ in $\mathrm{E} 7$ and $5 \mathrm{CB}$ and $3 \mathrm{wt} \%$ in $5 \mathrm{CB}$, were prepared by the following method. A total of $0.4 \mathrm{~mL}$ and $1.2 \mathrm{~mL}$ of AzoGNPs in toluene was added to $0.1 \mathrm{~g}$ of liquid crystals for $1 \mathrm{wt} \%$ and $3 \mathrm{wt} \%$ concentrations, respectively. The mixture was sonicated for several minutes to obtain a uniform blend, heated to $60^{\circ} \mathrm{C}$, and left overnight under an extraction hood to evaporate the solvent. The same technique was used to prepare a suspension of $1 \mathrm{wt} \%$ SPS nanoparticles in LC18523.

\subsection{Cell Preparation}

All measurements were performed using planar liquid crystal cells. The cells consisted of a thin layer of pure/undoped LC or nanoparticle suspensions between two glass slides. In the case of pure and doped LC18523, the cells studied in this paper were prepared ten years ago by our group. The gap between glass slides was fixed using $12 \mu \mathrm{m}$ spacers. The inner surfaces of the glass slides were covered with a conductive ITO layer and a rubbed polyimide alignment layer to obtain a planar orientation of the liquid crystal inside the cells. The LC18523 cells were stored in air at constant room temperature; all new measurements in this study were performed on the cells without any reheating, remixing or sonication. For the pure and doped E7 and 5CB cells, commercial planar cells purchased from EHC Co., Ltd. (Hachioji-shi, Tokyo) were used, with an ITO-coated surface of $50 \mathrm{~mm}^{2}$, polyimide alignment layers and a set gap between the glass slides of $10 \pm 0.5 \mu \mathrm{m}$.

All these cells were filled with either undoped LCs or suspensions in an isotropic phase using capillary forces and sealed using an epoxy glue to avoid leakage and contamination of the LC over time. Good-optical-quality liquid crystal alignment and suspension homogeneity across the cell were obtained. All measurements were performed at room temperature, approximately $22^{\circ} \mathrm{C}$, where the pure and doped LCs were in the nematic phase.

\subsection{CPI and Viscosity Measurements}

To measure and characterise our samples, we used a CPI setup [34] consisting of a planar liquid crystal cell placed between two crossed polarisers (polariser and analyser), with the LC director field at an angle of $45^{\circ}$ with the axes of polarisers. The cell was illuminated by a laser with a beam area of about $7 \mathrm{~mm}^{2}$ that defined the studied area of the samples. Two photodiodes recorded the light intensity before the polariser and after the analyser. We used $642 \mathrm{~nm}$ and $532 \mathrm{~nm}$ fibre-coupled diode lasers as beam sources. The schematic drawing of the setup is presented in Figure 1.

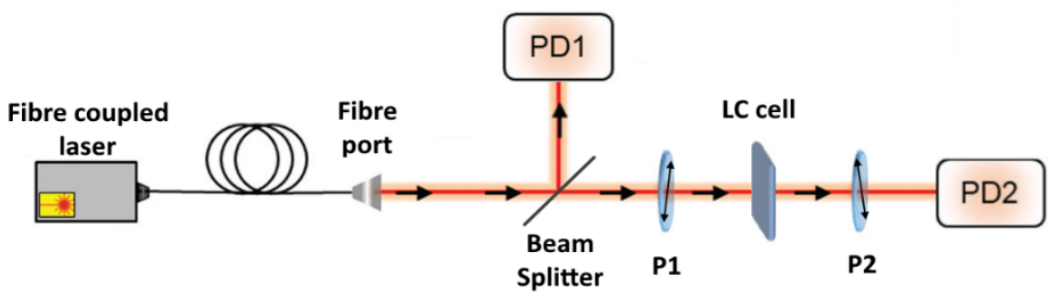

Figure 1. Experimental OMPA setup. A fibre-coupled diode laser beam is split so a portion of the light is directed to a first photodiode PD1 (reference signal), and the other part passes through the first polariser P1, the LC cell, and the second polariser P2 (analyser) before reaching the second photodiode PD2. The polariser and analyser axes are perpendicular to each other. This entire setup is enclosed to avoid any background noise.

This setup was controlled by a software, which performs data acquisition and analysis [34]. The cells were addressed with a sinusoidal AC signal at $10 \mathrm{kHz}$, the amplitude of which varied from 0 to $10 \mathrm{~V}$ with a voltage step of $0.02 \mathrm{~V}$. The average value of the CPI was measured at each voltage step. 
OMPA built-in algorithms fit CPI traces to determine elastic constants, cell thickness and pretilt angles. However, the dielectric constants and refractive indices of the suspensions should be specified for the numerical model to run correctly. The refractive indices were obtained from the literature $[14,35,38]$. Dielectric constants were obtained by measuring the capacitance of LC cells before and after filling them with the suspension using an automatic precision bridge (B905) from Wayne Kerr. The parallel component of the dielectric tensor, $\varepsilon_{/ /}$, was measured using a cell with homeotropic alignment, while the perpendicular component, $\varepsilon_{\perp}$, was obtained using a planar cell.

Viscosity measurements were carried out using the same CPI setup, following a method reported by us previously [39]. The cell was addressed with a high-frequency $(10 \mathrm{kHz})$-fixed voltage that had a low-frequency amplitude modulation. The frequency of the amplitude modulation varied between 0.1 and $10 \mathrm{~Hz}$. The driving voltage was selected to have a value above the Fredericks threshold, such that the CPI was around 50\% of the maximum value. The modulation amplitude was selected so the CPI oscillated between $20 \%$ and $80 \%$. We measured the standard deviation of the CPI oscillations, std(CPI), as a function of the frequency of the amplitude modulation. At low-amplitude modulation frequency, the CPI follows the variation of the driving signal amplitude. However, when the frequency of the amplitude modulation increases, the std(CPI) variation decreases as the LC is not able to follow the driving signal. OMPA fits the slope of the std(CPI) curve, evaluating the rotation viscosity of the LC suspension [39].

The OMPA set-up, in principle, can map a large area of a cell, determined by the size of the illuminating beam and the size of the ITO region [40]. However, in case of commercial cells, the limited size of the electrode did not allow us to probe the cells at different points across the cells.

\section{Results and Discussion}

In this section, we present OMPA measurements of elastic constants and rotational viscosity of nematic LCs E7 and 5CB doped with azo-thiolated gold nanoparticles and LC18523 doped with ferroelectric nanoparticles (SPS).

\subsection{Elastic Constants of AzoGNPs-Doped Liquid Crystals}

Azo-thiol gold nanoparticles (AzoGNPs) were synthesised by the method described in Section 2.1. We selected LCs that have been well studied in the literature as a base for the LC AzoGNPs suspensions, namely E7 and 5CB [9,38,41]. The suspensions with a particle content of $1 \mathrm{wt} \%$ AzoGNPs in both E7 and 5CB and $3 \mathrm{wt} \%$ AzoGNPs in 5CB were prepared. These amounts were chosen taking into account the relative stability of the NPs in the liquid crystal suspension, with the aim of having stable, well-dispersed NPs. Azo-GNP dispersed in nematic LCs have, so far, only been studied in 5CB, a single-component liquid crystal [20]. Here, we expanded this study to include Azo-GNP suspensions in E7 liquid crystal, a mixture of several mesogenic molecules. In addition, we investigated nematic LCs with ferroelectric nanoparticles, LC18523 liquid crystal doped with $1 \mathrm{wt} \%$ SPS. This suspension has already been explored in our previous work [35]. In the current study, we aimed to explore the stability of LC18523 + 1\%SPS over time, testing the cells prepared more than ten years ago.

As a first step, we investigated how elastic constants and viscosity change in LCs doped with NPs. The results of experimental CPI curves, and their fitting obtained by OMPA for 5CB-based AzoGNPs suspensions, are shown in Figure 2. The measured characteristics of all suspensions are summarised in Table 1. We can see from Figure 2 that the fits obtained by the OMPA match the experimental data minima and maxima very well. This ensures a precise estimation of both $\mathrm{K}_{1}$ and $\mathrm{K}_{3}$ [40]. The fit for doped E7 is available in the supporting information (Figure S1), also showing a good match of the minima and maxima with the experimental data. From these measurements, we can see that the OMPA is perfectly able to model stable LC-nanoparticle composites. 


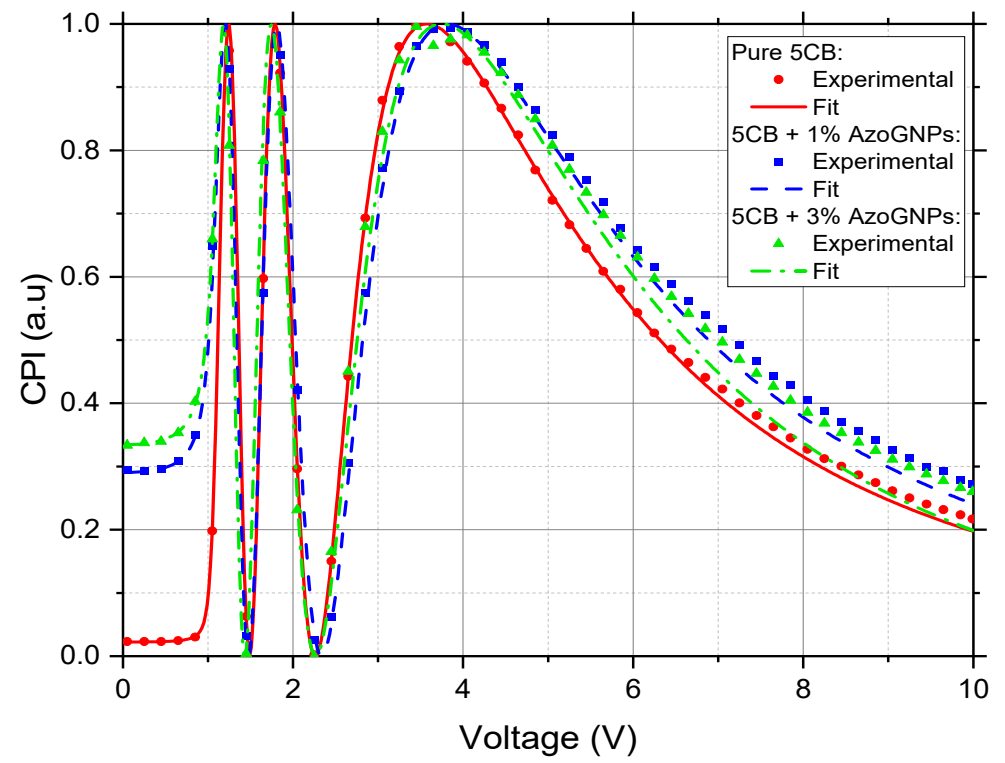

Figure 2. CPI measurements of pure and AzoGNP-doped 5CB under light illumination at $642 \mathrm{~nm}$ and a frequency of an applied electric field of $10 \mathrm{kHz}$. Experimental data are represented by symbols, the OMPA fits by lines.

Table 1. Elastic constants and rotational viscosity of pure and nanoparticle-doped E7, 5CB and LC18523 obtained through OMPA fitting. The changes of the dielectric anisotropy through doping are also shown: $\Delta \varepsilon$ decreased with the addition of AzoGNPs but increased with ferroelectric nanoparticles. The errors on the OMPA fits were studied previously for elastic constants [40] and are estimated to be approximately $1 \%$ for $\mathrm{K}_{1}$ and $2 \%$ for $\mathrm{K}_{3}$. The same has been carried out for viscosity measurements [38], and the error was estimated in the range of $1 \%$.

\begin{tabular}{|c|c|c|c|c|c|c|c|}
\hline & $\lambda(\mathrm{nm})$ & $\mathrm{K} 1(\mathrm{pN})$ & $\mathrm{K} 3(\mathrm{pN})$ & $\varepsilon_{/ /}$ & $\varepsilon_{\perp}$ & $\Delta \varepsilon$ & $\gamma_{1}$ (mPa.s) \\
\hline $5 \mathrm{CB}$ & 642 & 6.0 & 9.0 & 17.9 & 6.5 & 11.4 & 92.50 \\
\hline $5 \mathrm{CB}+1 \%$ AzoGNPs & 642 & 6.3 & 9.3 & 21.8 & 11.4 & 10.4 & 98.41 \\
\hline $5 \mathrm{CB}+3 \%$ AzoGNPs & 642 & 4.1 & 7.5 & 24.3 & 17.5 & 6.8 & 89.03 \\
\hline E7 & 642 & 10.7 & 16.2 & 19.5 & 5.2 & 14.4 & 203 \\
\hline $\mathrm{E} 7+1 \% \mathrm{AzoGNP}_{\mathrm{S}}$ & 642 & 8.9 & 16.6 & 17.6 & 7.5 & 10.1 & 148.17 \\
\hline LC18523 & 532 & 8.2 & 9.1 & 6.7 & 4.2 & 2.5 & 92.7 \\
\hline LC18523 + 1\%SPS & 532 & 6.5 & 11.0 & 11.5 & 4.7 & 6.8 & 128.58 \\
\hline
\end{tabular}

From Table 1, we noticed that the splay elastic constant $\mathrm{K}_{1}$ and dielectric permittivity $\Delta \varepsilon$ tended to decrease by $17 \%$ and $30 \%$ in E7 $+1 \%$ AzoGNPs and by $32 \%$ and $40 \%$ in $5 \mathrm{CB}+3 \%$ AzoGNPs, respectively. As reported in the literature, the process of particle aggregation may cause this behaviour [42]. Nanoparticles tended to disturb LC orientation in their neighbourhood. In the case of GNPs, the LC molecules interacted with the ligands on the surface of GNPs that locally distort LC orientation. This local distortion of LC around nanoparticles was more prominent for larger particles or as particle aggregation occurred. The small-size nanoparticles, however, can be incorporated into the LC matrix with minimum distortion. We noticed that the distortion of LC ordering can be observed by a decrease in elastic constants, as well as in dielectric anisotropy with doping. We can see that by increasing the concentration of nanoparticles, the values of the parallel and perpendicular components of dielectric tensor tended to increase; however, the difference between them decreased as the orientation became less uniform. As shown here, this was not the case for the $5 C B+1 \%$ AzoGNPs cell elastic constant, which increased by $5.5 \%$. We 
hypothesize that the aggregation process did not occur for this concentration, which agrees with the absence of particle precipitation at the bottom of a vial.

A similar behaviour in dielectric anisotropy and elastic constants was reported in the literature. Vardanyan et al. reported different doping concentrations of GNPs in 5CB [33,42]. They showed that both elastic constants and the dielectric anisotropy tend to decrease above a critical concentration, where gold aggregates start to form. They also discussed the increase in elastic constants, when the doping content is below this critical value, relating it to the formation of LC-gold conglomerates. The influence of GNPs content on the electrical, elastic and rheological properties of a nematic liquid crystal has also been studied by Chausov et al. [16]. Using gold nanoparticles of size between 7 and $15 \mathrm{~nm}$, they doped ZhK-1289 LC up to $5 \mathrm{wt} \%$. They reported an increase in the splay elastic constant and rotational viscosity at a low doping concentration $(<1 \mathrm{wt} \%)$ followed by a decrease in these parameters as the GNPs concentration increased. This trend is similar to what we observed in $5 \mathrm{CB}$, with $\mathrm{K}_{1}$ and $\gamma_{1}$ increasing in the AzoGNPs suspension with the doping content of $1 \mathrm{wt} \%$ and then decreasing in the suspension with $3 \mathrm{wt} \%$ AzoGNPs concentration. However, both these groups reported an increase in $\Delta \varepsilon$ at low concentrations of GNPs, which is not in agreement with our study of AzoGNPs.

In the case of ferroelectric nanoparticles, $\Delta \varepsilon$ increased 2.7 times while $K_{1}$ decreased by $20 \%$. Compared to the small GNPs (5 $\mathrm{nm}$ core), SPS nanoparticles are much larger (50 $\mathrm{nm}$ ) and thus cause a stronger distortion of the LCs around them even if they are well dispersed, leading to a decrease in $\mathrm{K}_{1}$. In contrast, the huge increase in dielectric anisotropy can be explained by the polarizability of ferroelectric inclusions, as reported before $[14,43,44]$.

\subsection{Rotational Viscosity Measurements}

Another key parameter of LCs is rotational viscosity $\gamma_{1}$, which determines the response time of LC devices. The viscosity of a liquid crystal can be significantly influenced by doping with nanoparticles. It has been reported that the viscosity of such colloidal system tends to increase for small amounts of dopants and to decrease when nanoparticles start to aggregate [33,42].

The rotational viscosity was measured using OMPA as described in Section 2.3. The experimental data were fitted with the OMPA algorithm using rotational viscosity as a fitting parameter [39]. The experimental data and fits for pure and doped 5CB are shown in Figure 3, and values for rotational viscosity for all suspension are given in Table 1 . We can observe from Figure 3 that the fit and the experimental data match the slope part of the CPI very well, which was then used by the model to estimate the rotational viscosity $\gamma_{1}$ [37]. This, once again, demonstrates that the OMPA model can fit nanoparticle-doped LCs.

The rotational viscosity increased from $92.5 \mathrm{mPa}$.s for undoped to $98.41 \mathrm{mPa}$.s for $5 \mathrm{CB}$ $+1 \%$ AzoGNPs. However, with the further increase in AzoGNPs content in the suspension to $3 \%$, the rotational viscosity dropped to $89.03 \mathrm{mPa}$.s. This observation could be explained by the formation of irregular-shaped aggregates that disturb the LCs in the surroundings, as the concentration of AzoGNPs increases. The presence of gold nanoparticle aggregates tends to lower the viscosity of the suspension as the liquid crystal has more space to move freely, whereas well-dispersed GNPs may interfere with the reorientation process [6,42].

The rotational viscosity fit for E7 $+1 \mathrm{wt} \%$ AzoGNPs can be found in the supporting information (Figure S2), also showing a good agreement with the experimental data. For this suspension, we observed that the rotational viscosity decreased from $203 \mathrm{mPa}$.s for the undoped E7 to $148.17 \mathrm{mPa} . \mathrm{s}$ in the suspension with $1 \%$ of nanoparticle content. Such a trend indicates the presence of particle aggregates in the suspension. No studies were carried out on E7 + 3\%AzoGNPs as it was not possible to obtain a stable, colloidal suspension at this concentration. 


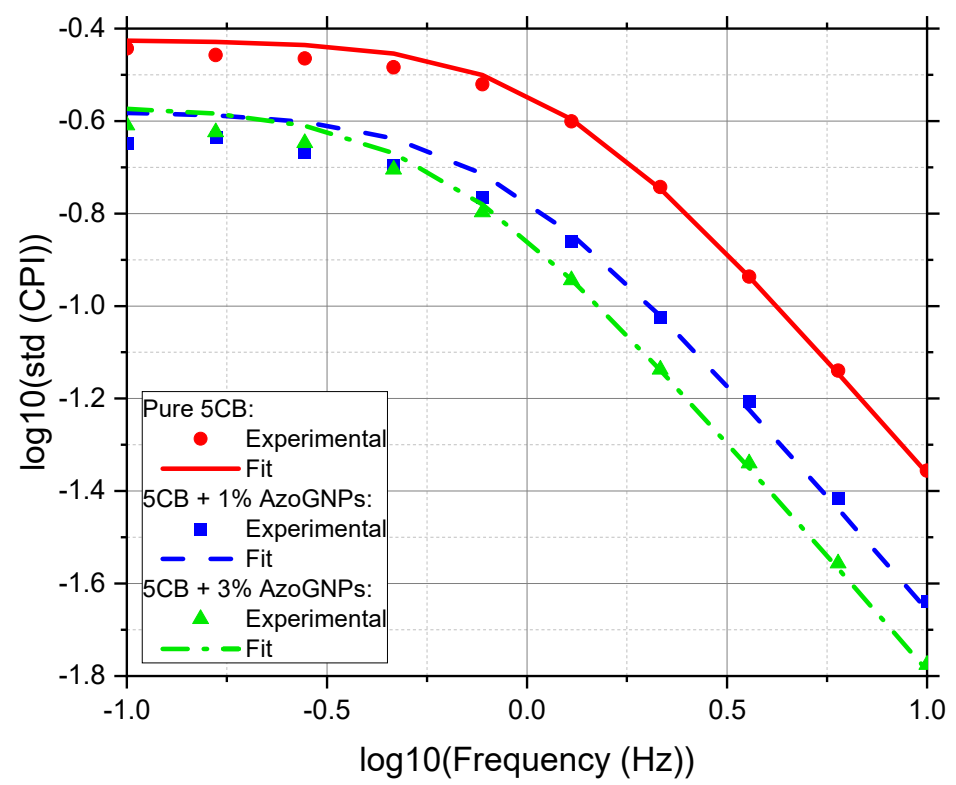

Figure 3. Rotational viscosity measurements of pure and nanoparticle-doped 5CB under light illumination at $642 \mathrm{~nm}$. Experimental data are represented by symbols, and the OMPA fits are shown by solid lines.

For the LC18523 + SPS suspension, we obtained an increase in the viscosity by a factor of 1.4 in comparison with the undoped LC18523. In contrast to AzoGNPs, the interaction between SPS NPs and LC molecules is mainly due to the strong dipole moment of the nanoparticles. This strong interaction induces local areas where LC molecules are attracted to the nanoparticles, and thus are more difficult to reorient, increasing the average viscosity of the suspension.

This suspension was studied by us earlier in [35], where we estimated the rotation viscosity by measuring a response time of a twist cell. The previous results suggest that doping with $1 \mathrm{wt} \%$ of SPS increased the rotational viscosity by a factor of 3.2. Hence, we suggest that some degree of particle aggregation has occurred over time. As discussed earlier, the increase in the degree of particle aggregation can lead to the decrease in the rotational viscosity in the suspension from a factor of 3.2 [35] to a lower value, as seen in the current study, in which our repeated measurements showed a value of 1.4. However, we can still observe that the rotational viscosity of the doped LC18523 remains higher than that of the undoped version of this liquid crystal, meaning that the aggregation process is not solely responsible for the increased viscosity observed.

\subsection{Elastic Constants and Stability of Ferroelectric Nanoparticle-Doped LCs}

In this section we further analyse the stability of the SPS colloidal suspension. To do so, we compared OMPA fits obtained using experimental data measured previously for LC18523 and LC18523 + $1 \mathrm{wt} \%$ SPS suspensions [35], with new measurements of the same suspension. The CPI traces in [35] were measured using a laser beam at $633 \mathrm{~nm}$, and the new experiments were performed using a wavelength of $532 \mathrm{~nm}$. Fitting the previous data at $633 \mathrm{~nm}$ allowed us to compare our results with the values from the literature [35] and ensure the precision and capacity of the OMPA model in characterising such colloidal LC systems $[45,46]$. By repeating this measurement using the same samples, we checked the stability of the suspension over time. The results are summarised in Table 2 and shown on Figure 4 . 
Table 2. Summary of elastic constants obtained by the OMPA through the fitting of CPIs from cells filled with pure and nanoparticle-doped LC18523. These cells were measured using light illumination at $633 \mathrm{~nm}$ and $532 \mathrm{~nm}$.

\begin{tabular}{cccc}
\hline & $\boldsymbol{\lambda}(\mathbf{n m})$ & $\mathbf{K 1}(\mathbf{p N})$ & $\mathbf{K 3}(\mathbf{p N})$ \\
\hline Pure LC18523 (literature [35]) & 633 & 7.85 & 10.0 \\
\hline Pure LC18523 (OMPA fit of [35]) & 633 & 7.9 & 10.1 \\
\hline $\begin{array}{c}\text { Pure LC18523 (OMPA new } \\
\text { measurements) }\end{array}$ & 532 & 8.2 & 9.1 \\
\hline LC18523 + 1 wt\% SPS (literature [35]) & 633 & 7.1 & 10.5 \\
\hline LC18523 + 1 wt\% SPS (OMPA fit of [35]) & 633 & 7.3 & 10.5 \\
\hline $\begin{array}{c}\text { LC18523 + 1 wt\% SPS (OMPA new } \\
\text { measurements) }\end{array}$ & 532 & 6.5 &
\end{tabular}

(a)

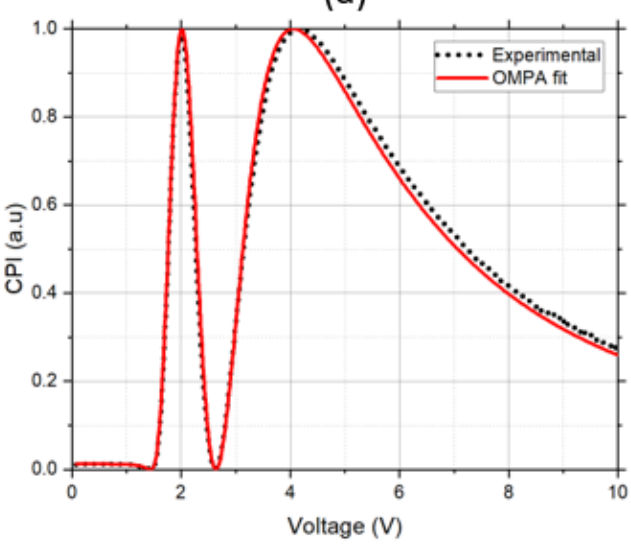

(b)

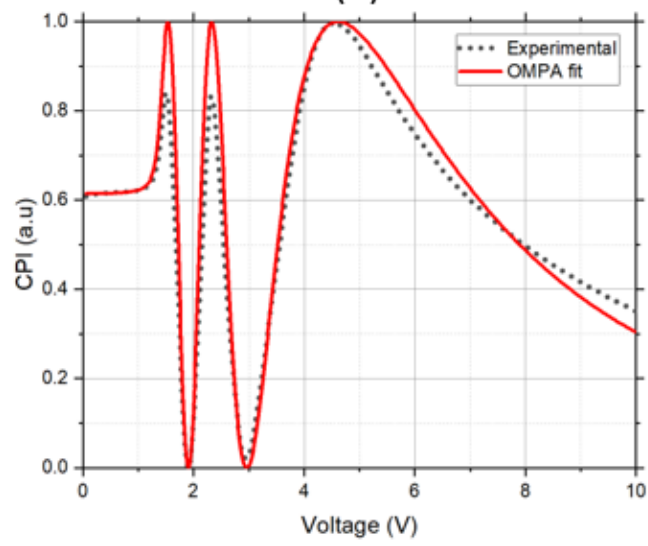

Figure 4. Experimental CPI measurements (black dots) and corresponding OMPA fit (solid red line) of LC18523 + 1 wt\% SPS at $633 \mathrm{~nm}(\mathbf{a})$ and $532 \mathrm{~nm}(\mathbf{b})$ at $10 \mathrm{kHz}$. The CPI data fitted at $633 \mathrm{~nm}$ (a) was collected and published previously [35], and the CPI data fitted at $532 \mathrm{~nm}$ (b) were measured recently. The fitted parameters that correspond to these fits are presented in Table 2.

From Figure 4 and Table 2, we can notice that OMPA provides a good-quality fit to the previously reported data at $633 \mathrm{~nm}$, and the values of the elastic constants show good agreement with the literature values [35]. However, we could not obtain the same goodquality fit for the repeated experiments at $532 \mathrm{~nm}$. This is also reflected in some deviation in the fitted values of the elastic constants in comparison to the elastic constants obtained at $633 \mathrm{~nm}$. The splay elastic constant $\mathrm{K}_{1}$ decreased from $7.1 \mathrm{pN}$ to $6.5 \mathrm{pN}$, while the bend elastic constant $\mathrm{K}_{3}$ increased from $10.5 \mathrm{pN}$ to $11 \mathrm{pN}$. This observation was reproducible across the cell.

The decrease in $K_{1}$ can be explained by the quality of the experimental CPI trace at low voltage, where the first two maximums had a reduced value (see Figure $4 \mathrm{~b}$ ), leading to an underestimation of $K_{1}$ during fitting [40]. As the samples were old, we believe that this is another sign that some degree of aggregation occurred in the suspension. The aggregates caused nonuniform liquid crystal orientation around them. This effect became especially prominent at voltages around the threshold value, when LC orientation was not uniform across the cell. This nonuniformity in LC alignment resulted in an increase in light scattering at this voltage range, observed by the decrease in the height of the first CPI maximum. The increased value of $\mathrm{K}_{3}$ could be due to the lack of agreement between fitting and experimental curves at a high-voltage range, the part of CPI trace that is used to extract $\mathrm{K}_{3}[40]$.

As the model from the OMPA shows a very good agreement at $633 \mathrm{~nm}$ for both the fitted and literature values of $K_{1}$ and $K_{3}$, we can conclude that the OMPA parameters are 
reliable. The difference in the magnitude of the elastic constants for doped LC18523, from the current fitting and the previously published work, confirms the trend observed for the long-term rotational viscosity changes, suggesting that some degree of aggregation of nanoparticles is the underlying mechanism.

\section{Conclusions}

We have demonstrated that OMPA can successfully characterise nematic LCs doped with different nanoparticles, such as ferroelectric and gold nanoparticles. In particular, we prepared, and then characterised for the first time to our knowledge, a stable suspension of AzoGNPs in E7. Knowing only dielectric coefficients and refractive indices, we were able to extract both elastic constants and the rotational viscosity of pure and doped LCs, the parameters that are important for the electric field and time response. This result is, indeed, consistent with homogenisation studies of liquid crystal suspensions, which show that the suspension can be modelled by an effective theory, in which the LC crystal parameters are altered by the presence of the nanoparticles. The values of elastic constants and rotational viscosity were also used to capture the long-term stability and ageing changes in the cells, as in the case investigated here, identifying the process of aggregation in colloidal suspensions of ferroelectric nanoparticles, such as LC18523 doped with SPS nanoparticles. Therefore, we showed that OMPA is a very versatile and useful tool able to characterise not only nematic single components and mixtures but also doped LC systems.

Supplementary Materials: The following supporting information can be downloaded at: https: //doi.org/10.5258/SOTON/D2106, Figure S1: CPI measurements vs. OMPA fit of E7 + $1 \mathrm{wt} \%$ AzoGNPs at $642 \mathrm{~nm}$ and $10 \mathrm{kHz}$. Figure S2: Rotational viscosity CPI measurements vs. OMPA fit of $\mathrm{E} 7+1 \mathrm{wt} \%$ AzoGNPs at $642 \mathrm{~nm}$.

Author Contributions: Experiments and data analysis, N.B.; sample preparation N.P. and T.O.; material synthesis A.F.D.F. and A.G.K.; software, N.P. and G.D.; error propagation, D.B. and G.D.; writing-original draft preparation, N.B. and N.P.; M.K. and G.D. proposed the initial idea and together with O.H., who supervised the project. All authors have contributed to reviewing and editing the published version of the manuscript. All authors have read and agreed to the published version of the manuscript.

Funding: This work was supported by the Leverhulme Trust (grant RPG-2019-055).

Institutional Review Board Statement: Not applicable.

Informed Consent Statement: Not applicable.

Data Availability Statement: The data sets generated and analysed during the current study are available from the corresponding author on reasonable request.

Conflicts of Interest: The authors declare no conflict of interest.

\section{References}

1. Shen, Y.; Dierking, I. Perspectives in Liquid-Crystal-Aided Nanotechnology and Nanoscience. Appl. Sci. 2019, 9, 2512. [CrossRef]

2. Munna, M.; Anwar, F.; Coutu, R.A. Nematic Liquid Crystal Composite Materials for DC and RF Switching. Technologies 2019, 7, 32. [CrossRef]

3. Kumar, A.; Singh, D.P.; Singh, G. Recent progress and future perspectives on carbon-nanomaterial-dispersed liquid crystal composites. J. Phys. D Appl. Phys. 2022, 55, 38. [CrossRef]

4. Ayeb, H.; Alaya, S.; Derbali, M.; Samet, L.; Bennaceur, J.; Jomni, F.; Soltani, T. Dielectrical, electro-optical and textural studies of 5CB nematic liquid crystal doped with $\mathrm{TiO} 2$ and $\mathrm{Cu}-\mathrm{TiO}_{2}$ nanoparticle. Liq. Cryst. 2021, 48, 223-232. [CrossRef]

5. Mirzaei, J.; Urbanski, M.; Kitzerow, H.S.; Hegmann, T. Synthesis of liquid crystal silane-functionalized gold nanoparticles and their effects on the optical and electro-optic properties of a structurally related nematic liquid crystal. Chemphyschem 2014, 15, 1381-1394. [CrossRef]

6. Roy, A.; Singh, B.P.; Yadav, G.; Khan, H.; Kumar, S.; Srivastava, A.; Manohar, R. Effect of gold nanoparticles on intrinsic material parameters and luminescent characteristics of nematic liquid crystals. J. Mol. Liq. 2019, 295, 111872. [CrossRef]

7. Sano, S.; Miyama, T.; Takatoh, K.; Kobayashi, S. Enhancement of the characteristics of LCDs by doping nanoparticles: Reduction of the operating voltage, viscosity, and response times. In Proceedings of the SPIE 6135, Liquid Crystal Materials, Devices, and Applications XI, 613501. Event: Integrated Optoelectronic Devices 2006, San Jose, CA, USA, 23 February 2006. [CrossRef] 
8. Tripathi, P.K.; Misra, A.K.; Manohar, S.; Gupta, S.K.; Manohar, R. Improved dielectric and electro-optical parameters of ZnO nano-particle $\left(8 \% \mathrm{Cu}^{2+}\right)$ doped nematic liquid crystal. J. Mol. Struct. 2013, 1035, 371-377. [CrossRef]

9. Pandey, A.S.; Dhar, R.; Kumar, S.; Dabrowski, R. Enhancement of the display parameters of $4^{\prime}$-pentyl-4-cyanobiphenyl due to the dispersion of functionalised gold nano particles. Liq. Cryst. 2011, 38, 115-120. [CrossRef]

10. Singh, U.B.; Dhar, R.; Dabrowski, R.; Pandey, M.B. Enhanced electro-optical properties of a nematic liquid crystals in presence of BaTiO3 nanoparticles. Liq. Cryst. 2014, 41, 953-959. [CrossRef]

11. Garbovskiy, Y.; Glushchenko, A. Ferroelectric Nanoparticles in Liquid Crystals: Recent Progresss and Current Challenges Nanomaterials 2017, 7, 361. [CrossRef]

12. Al-Zangana, S.; Turner, M.; Dierking, I. A comparison between size dependent paraelectric and ferroelectric BaTiO3 nanoparticle doped nematic and ferroelectric liquid crystals. J. Appl. Phys. 2017, 121, 085105. [CrossRef]

13. Evans, D.R.; Cook, G.; Reshetnyak, V.Y.; Liebig, C.M.; Basun, S.A.; Banerjee, P.P. Inorganic-Organic Photorefractive Hybrids In Photorefractive Organic Materials and Applications; Springer Series in Materials Science; Blanche, P.A., Ed.; Springer: Cham, Switzerland, 2016; Volume 240. [CrossRef]

14. Kaczmarek, M.; Buchnev, O.; Nandhakumar, I. Ferroelectric nanoparticles in low refractive index liquid crystals for strong electro-optic response. Appl. Phys. Lett. 2008, 92, 87. [CrossRef]

15. Lena, M.; Lopatina; Jonathan, V. Maier-Saupe-type theory of ferroelectric nanoparticles in nematic liquid crystals. Selinger Phys. Rev. 2011, 84, 041703.

16. Chausov, D.N.; Kurilov, A.D.; Kucherov, R.N.; Simakin, A.V.; Gudkov, S.V. Electro-optical performance of nematic liquid crystals doped with gold nanoparticles. J. Phys. Condens. Matter 2020, 32, 395102. [CrossRef]

17. Acreman, A.; Kaczmarek, M.; D'Alessandro, G. Gold nanoparticle liquid crystal composites as a tunable nonlinear medium. Phys. Rev. 2014, 90, 012504. [CrossRef] [PubMed]

18. Lysenko, D.; Ouskova, E.; Ksondzyk, S.; Reshetnyak, V.; Cseh, L.; Meh, G.H.; Reznikov, Y. Light-induced changes of the refractive indices in a colloid of gold nanoparticles in a nematic liquid crystal. Eur. Phys. J. E 2012, 35, 33. [CrossRef] [PubMed]

19. Podoliak, N.; Bartczak, D.; Buchnev, O.; Kanaras, A.G.; Kaczmarek, M. High Optical Nonlinearity of Nematic Liquid Crystals Doped with Gold Nanoparticles. J. Phys. Chem. C 2012, 116, 12934-12939. [CrossRef]

20. Xue, C.; Xiang, J.; Nemati, H.; Gutierrez-Cuevas, H.K.B.K.; Wang, L.; Gao, M.; Zhou, S.; Yang, D.; Lavrentovich, O.D.; Urbas, A.; et al. Light-Driven Reversible Alignment Switching of Liquid Crystals Enabled by Azo Thiol Grafted Gold Nanoparticles. ChemPhysChem 2015, 16, 1852-1856. [CrossRef] [PubMed]

21. Milette, J.; Toader, V.; Reven, L.; Lennox, R.B. Tuning the miscibility of gold nanoparticles dispersed in liquid crystals via the thiol-for-DMAP reaction. J. Mater. Chem. 2011, 21, 9043. [CrossRef]

22. Mirzaei, J.; Reznikov, M.; Hegmann, T. Quantum dots as liquid crystal dopants. J. Mater. Chem. 2012, 22, 22350-22365. [CrossRef]

23. Singh, U.B.; Dhar, R.; Pandey, A.S.; Kumar, S.; Dabrowski, R.; Pandey, M.B. Electro-optical and dielectric properties of CdSe quantum dots and 6CHBT liquid crystals composites. AIP Adv. 2014, 4, 117112. [CrossRef]

24. Konshina, E.A.; Galin, I.F.; Shcherbinin, D.P.; Gavrish, E.O. Study of dynamics and relaxation optical response of nematic liquid crystals doped with CdSe/ZnS quantum dots. Liq. Cryst. 2014, 41, 1229-1234. [CrossRef]

25. Gupta, S.K.; Singh, D.P.; Tripathi, P.K.; Manohar, R.; Varia, M.; Sagar, L.K.; Kumar, S. CdSe quantum dot-dispersed DOBAMBC: An electro-optical study. Liq. Cryst. 2013, 40, 528-533. [CrossRef]

26. Rastogi, A.; Pathak, G.; Srivastava, A.; Herman, J.; Manohar, R. Cd1-X ZnXS/ZnS core/shell quantum dots in nematic liquid crystals to improve material parameter for better performance of liquid crystal-based devices. J. Mol. Liq. 2018, 255, 93-101. [CrossRef]

27. Satapathy, P.; Vasudeva, N.; Oinam, J.; Prasad, S.K. Enhanced luminescence, electric-field and actinic-light modulation of emission in nematic-CdSeS gradient nanocrystal composites by polymer confinement. J. Mol. Liq. 2021, 46, 1595-1604. [CrossRef]

28. Satapathy, P.; Santra, P.K.; Haque, A.; Yelamaggad, C.V.; Das, S.; Prasad, S.K. Anisotropic Fast Electrically Switchable Emission from Composites of CsPbBr3 Perovskite Quantum Cuboids in a Nematic Liquid Crystal. Adv. Opt. Mater. 2019, 7, 1801408. [CrossRef]

29. Joshi, T.; Kumar, A.; Prakash, J.; Biradar, A.M. Low power operation of ferroelectric liquid crystal system dispersed with zinc oxide nanoparticles. Appl. Phys. Lett. 2010, 96, 253109. [CrossRef]

30. Podoliak, N.; Buchnev, O.; Buluy, O.; D’Alessandro, G.; Kaczmarek, M.; Reznikov, Y.; Sluckin, T.J. Macroscopic optical effects in low concentration ferronematics. Soft Matter 2011, 7, 4742-4749. [CrossRef]

31. Tóth-Katona, T.; Gdovinová, V.; Tomašovičová, N.; Éber, N.; Fodor-Csorba, K.; Juríková, A.; Závišová, V.; Timko, M.; Chaudc, X.; Kopčanský, P. Tuning the phase transition temperature of ferronematics with a magnetic field. Soft Matter 2018, 14, 1647-1658. [CrossRef] [PubMed]

32. Gdovinová, V.; Schroer, M.A.; Tomašovičová, N.; Appel, I.; Behrens, S.; Majorošová, J.; Kováč, J.; Svergunb, D.I.; Kopčanskýa, P. Structuralization of magnetic nanoparticles in 5CB liquid crystals. Soft Matter 2017, 13, 7890-7896. [CrossRef]

33. Vardanyan, K.K.; Sita, D.M.; Walton, R.D.; Saidel, W.M.; Jones, K.M. Cyanobiphenyl liquid crystal composites with gold nanoparticles. RSC Adv. 2013, 3, 259. [CrossRef]

34. Perivolari, E.; D'Alessandro, G.; Apostolopoulos, V.; Brouckaert, N.; Heiser, T.; Kaczmarek, M. Two-dimensional snapshot measurement of surface variation of anchoring in liquid crystal cells. Liq. Cryst. 2021, 48, 2086-2096. [CrossRef] 
35. Podoliak, N.; Buchnev, O.; Herrington, M.; Mavrona, E.; Kaczmarek, M.; Kanaras, A.G.; Stratakis, E.; Blach, J.F.; Henninot, J.F.; Warenghem, M. Elastic constants, viscosity and response time in nematic liquid crystals doped with ferroelectric nanoparticles. RSC Adv. 2014, 4, 46068-46074. [CrossRef]

36. Brust, M.; Schiffrin, D.J.; Bethell, D.; Kiely, C.J. Novel gold-dithiol nano-networks with non-metallic electronic properties. Adv. Mater. 1995, 7, 795-797. [CrossRef]

37. Herrington, M.R.; Buchnev, O.; Kaczmarek, M.; Nandhakumar, I. The Effect of the Size of BaTiO3 Nanoparticles on the ElectroOptic Properties of Nematic Liquid Crystals. Mol. Cryst. Liq. Cryst. 2010, 527, 72-228. [CrossRef]

38. Demus, D.; Inukai, T. Calculation of molecular, dielectric and optical properties of 4'-n-pentyl-4-cyano-biphenyl (5CB). Liq. Cryst. 1999, 26, 1257-1266. [CrossRef]

39. Bennett, T.P.; Proctor, M.B.; Kaczmarek, M.; D'Alessandro, G. Lifting degeneracy in nematic liquid crystal viscosities with a single optical measurement. J. Colloid Interface Sci. 2017, 497, 201-206. [CrossRef]

40. Bennett, T.; Proctor, M.; Forster, J.; Perivolari, E.; Podoliak, N.; Sugden, M.; Kirke, R.; Regrettier, T.; Heiser, T.; Kaczmarek, M.; et al. Wide area mapping of liquid crystal devices with passive and active command layers. Appl. Opt. 2017, 56, 9050-9056. [CrossRef] [PubMed]

41. Cui, M.; Kelly, J.R. Temperature Dependence of Visco-Elastic Properties of 5CB. Mol. Cryst. Liq. Cryst. Sci. Technol. Sect. A. Mol. Cryst. Liq. Cryst. 1999, 331, 49-57. [CrossRef]

42. Vardanyan, K.K.; Sita, D.M.; Walton, R.D.; Gurfinkiel, I.S.; Saidel, W.M. Liquid crystalline cyanobiphenyl homologues doped with gold nanoparticles. Liq. Cryst. 2012, 39, 1083-1098. [CrossRef]

43. Reznikov, Y.; Buchnev, O.; Tereshchenko, O.; Reshetnyak, V.; Glushchenko, A.; West, J. Ferroelectric nematic suspension. Appl. Phys. Lett. 2003, 82, 1917-1919. [CrossRef]

44. Li, F.; Buchnev, O.; Cheon, C.; Glushchenko, A.; Reshetnyak, V.; Reznikov, Y.; Sluckin, T.J.; West, J.L. Orientational coupling amplification in ferroelectric nematic colloids. Phys. Rev. Lett. 2006, 97, 147801. [CrossRef]

45. Bennett, T.P.; D'Alessandro, G.; Daly, K.R. Multiscale models of colloidal dispersion of particles in nematic liquid crystals. Phys. Rev. 2014, 90, 062505. [CrossRef] [PubMed]

46. Bennett, T.P.; D'Alessandro, G.; Daly, K.R. Multiscale Models of Metallic Particles in Nematic Liquid Crystals. SIAM J. Appl. Math. 2018, 78, 1228-1255. [CrossRef] 